\title{
The upper cervical spine tumor pathology C1-C2 - therapeutic attitude
}

\author{
D. Serban, N.A. Calina, Fl. Exergian, M. Podea, C. Zamfir, E. Morosanu, \\ A. Giovani, Gh. Checiu
}

Spinal Surgery Department, First Neurosurgical Clinic

Clinic Emergency Hospital "Bagdasar-Arseni”, Bucharest, Romania

\begin{abstract}
Surgical treatment of upper cervical spine tumors, whether they are vertebral, epidural, subdural or intramedullary, raises technical and decisional difficulties regarding the approach of the region as well as in maintaining its stability.

The authors performed a retrospective study on C1, C2 spinal tumor pathology, managed surgically in the Spinal Surgery Department of Bagdasar Arseni Clinical Hospital, between January 2007 and December 2011.

We included in the study 44 patients, operated for C1, C2 cervical spine tumors, 23 men and 21 women with ages between 13 and 71 years. The pathology included 24 C1-C2 vertebral tumors, 11 subdural tumors, 2 epidural tumors and 7 intramedullary tumors.

Presenting symptoms were cervical pain, occipital neuralgia, medullary compression syndrome, and/or cranio-spinal junction instability.

The purpose of surgery was to establish a histopathologic diagnosis and to decompress the neural elements by attempting a total tumor removal as well as to stabilize the cranio - cervical junction in order to improve the patient's quality of life. The approach was chosen based on tumor location, prognosis and the need for
\end{abstract}

fixation. For 6 patients an anterior approach was used, for 31 pacients we used a posterior approach and 7 patients required a combined anterior and posterior approach.

Neurological improvement was observed in 17 patients, with a mean increase of 8 points on ASIA scale, 7 patients worsened immediately postoperatively with a mean decrease of 10 points on ASIA scale, (2 patients died), and 20 patients without neurological deficits preoperatively remained unchanged. In all cases where the craniospinal junction instability was the cause of occipito-cervical pain we noted the disappearence of pain after surgery.

The development of new surgical techniques and fixation systems paved the way to a successful treatment for these difficult tumors, some of them considered inoperable in the past.

Key words: atlas, axis, cranio-spinal junction, tumor, cranio-spinal instability.

\section{Introduction}

Craniospinal junction tumors are rare lesions that have a natural history towards tetraplegia, usually being a serious threat to the life of the patient. The treatment of these upper cervical spine tumors involving C1 and C2 segments has improved considerably in the last decades. The 
imaging investigations that allow the precise definition of the anatomical structures involved in the tumor pathology together with refined surgical techniques and the more and more complex fixation instrumentations allow for the complete removal of craniospinal junction tumors with the preservation or the regaining of stability. $(1,2,3)$

\section{Clinical material and methods}

In this retrospective study we reviewed the medical files of all the patients operated for C1, C2 spinal tumoral pathology in the Spinal Surgery Department of "Bagdasar Arseni" Emergency Hospital. We analyzed the data regarding the age and sex of the patients, the location (vertebral body, posterior arch, etc) and type of the tumor: epidural, subdural or intramedullary. We also noted the surgical approach and the technical subtleties that we used for the tumor resection as well as the procedures used for occipito-cervical fixation, the histopathologic results and the neurological deficits evaluated according to the Frankel scale and the ASIA score, pre and postoperatively.

We included in the sudy only the patients operated in our department between January 2007 and December 2011. In 5 years we performed 5780 spinal procedures in our department. Of these surgeries $813(14 \%)$ were for tumors and of these 121 were performed for tumors with cervical location. Of the 52 patients admitted for tumors located at C1-C2 level only 44 were surgically treated and included in the study. The remaining 8 patients were inoperable. These 44 patients included in the study represent $36 \%$ of the tumoral lesions with cervical location and $0,7 \%$ of all spinal pathology operated in our department during the time span of the study.

There was an almost equal sex distribution, 23 men and 21 women, sex ratio $=1.095$. The patients were aged between 13 and 71 years old with a mean of 48 years. The incidence of cervical spine tumors was higher beginning with the 5 th decade, vertebral metastasis being the predominant type of tumors in the old age.

Almost all patients presented upper cervical pain at admission, 21 pacients were admitted with occipital neuralgia (50\%) and 29 pacients $(69 \%)$ presented neurological deficit as a consequence of medullary compression. Less common symptoms were sensory deficit, urinary incontinence or cranial nerves palsy when the tumor extended intracranially. The symptoms had an insidious onset in $37(84 \%)$ patients and in $7(16 \%)$ patients the symptoms appeared after injuries. 10 patients presented craniospinal junction instability preoperatively.

We included in the study all C1 and C2 vertebral tumors, primary or metastatic, epidural, subdural, intraforaminal and intramedullary.

The histopathology (table 1) showed a great diversity of these tumors, including:

- 24 vertebral tumors:

- 7 of these were primary bone tumors, represented by fibrous bone dysplasia, chondroid chordoma, osteoblastoma, sarcoma, chondroma, and bone giant cell tumor.

- 17 pacients presented metastases most of them with the primary tumor in the lungs.

- Out of 11 subdural tumors, 4 were schwanomas, 3 neurofibromas, and 4 meningiomas.

- 7 cases of intramedullary tumors, most of them ependymomas

- 2 epidural tumors 
DOI: $10.2478 / v 10282-012-0012-3$

TABLE 1

C1, C2 cervical tumoral pathology - histopathology types

\begin{tabular}{|c|c|c|c|c|c|c|c|}
\hline \multicolumn{2}{|l|}{$\begin{array}{l}\text { Vertebral tumors } \\
24 \text { cases }\end{array}$} & \multicolumn{2}{|c|}{\begin{tabular}{|l|l|} 
Subdural \\
extramedulary tumors; \\
“dumpbell” tumors \\
11 cases
\end{tabular}} & \multicolumn{2}{|l|}{$\begin{array}{l}\text { Intramedullary } \\
\text { tumors } \\
7 \text { cases }\end{array}$} & \multicolumn{2}{|l|}{\begin{tabular}{|l} 
Epidural \\
tumors
\end{tabular}} \\
\hline Tumor type & $\begin{array}{l}\text { Nr. } \\
\text { Pat. }\end{array}$ & Tumor type & Nr. Pat. & Tumor type & $\begin{array}{l}\text { Nr. } \\
\text { Pat. }\end{array}$ & Tumor type & $\begin{array}{l}\text { Nr. } \\
\text { Pat. }\end{array}$ \\
\hline $\begin{array}{l}\text { Metastasis } \\
\text { - renal carcinoma tumor } \\
\text { - breast } \\
\text { - pulmonary } \\
\text { - cheratinised squamocellular } \\
\text { - tyroidian } \\
\text { - uterine } \\
\text { - undifferentiated } \\
\end{array}$ & $\begin{array}{l}2 \\
3 \\
6 \\
1 \\
1 \\
1 \\
3\end{array}$ & Schwanoma & 4 & Ependimoma & 6 & Sarcom Ewing & 1 \\
\hline bone fibrous Dysplasia & 1 & Meningioma & 4 & Hemangioblastoma & 1 & \begin{tabular}{|l} 
Peripheral \\
nerve sheath \\
malignant \\
tumor.
\end{tabular} & 1 \\
\hline Chondroid Chordoma & 1 & Neurofibroma & 3 & & & & \\
\hline Osteoblastoma & 2 & & & & & & \\
\hline Sarcoma & 1 & & & & & & \\
\hline Chondroma & 1 & & & & & & \\
\hline Bone giant cell tumor & 1 & & & & & & \\
\hline
\end{tabular}

\section{Imaging studies}

Preoperative work-up included an MRI with minimum T1, T2 and T1 with contrast, useful both as a diagnostic tool and for surgical planning. In most situations plain X-rays and CT scans are required for a better visualization of bony structures, but the MRI is by far the most valuable investigation in this pathology. It is indispensable and usually sufficient in most subdural and intramedullary tumors.

CT scan is impractical for the screening of vertebral tumors, but extremely valuable for the preoperative planning. The occipitocervical vertebral CT with sagittal and coronal reconstructions helps to establish the tumor location and the extension of bone distruction.

Plain X-ray is a screening investigation but it wasn't used for surgical planning and decision. We used the dynamic flexion- extension X-ray to establish the degree of instability preoperatively and for the postoperative control of stability.

Patients with suspected multiple metastatic lesions had brain, thoracic and abdominal CT-scans done, in order to establish the extent of the disease and to estimate the life expectancy.

Bone scintigraphy is valuable in the diagnosis of vertebral tumors, but it lacks specificity (false positive results in infections and fractures).

AngioCT and spinal angiography are valuable for diagnosis and surgical planning and can be used for embolization of the tumor prior to surgery. We used these investigations in those cases where the tumor encased the vertebral arteries. Its greatest value stands in establishing the relationship between the tumor and the blood vessels, especially when the purpose 
of surgery is total resection. $(1,5)$

Tumor biopsy is reserved for cases which lack neurological signs or spinal instability. A reasonable enough indication for biopsy is the suspicion of a certain histopathological type of tumor that is essentially treatable by non surgical therapy (radio/chemotherapy or percutaneous vertebroplasty with poliacrilic cement, etc.)

\section{Therapeutic attitude in tumors localised at C1 and C2 level}

It is certain that not all situations can or must be solved using the same technique. However the surgical indication is the rule.

The surgical indications won't be discussed here. Though it must be said that there are histopathological subtypes which usually don't require surgery. For example, the eosinophilic granuloma which can be treated by simple immobilization or the lymphomas and plasmocytomas that are radio and/or chemotherapy sensitive.

The surgical decision was made after documenting the local or radicular pain (impossible to alleviate by medication), the instability of the craniospinal junction C1-C2 luxation in a tumor modified bone, neurological deficit, clinical and biological state of the pacient, age, related diseases, life expectancy, the presence of metastases in other locations in the spine or in other organs, primary tumor radiosensitivity. (2, $7,9,10)$

Absolute or relative contraindications were considered to be:

- complete neurological deficits

- excesive vital risk due to other severe diseases

- terminal stage neoplasia with multiple metastases and extended tumoral lesions in which achieving postoperative stabilization would be impossible.

Radio and/or chemotherapy can be used as treatment regimens alone or combined with surgery. This can be established after a precise histopatological diagnosis, tumor sensitivity and evaluation of test results.

Embolization can be used before surgery. Indications depend on the histopathological type of tumor (hemangioma, aneurismal cyst, other metastases), and on the degree of vascularization seen by spinal angiography and finally on the existance of technical means and of the necessary knowledge.

Autopolimeryzable cement transcutaneous (transoral) injection is quite a simple method indicated in elderly patients. This is a therapeutic solution only if the CT confirms an intact vertebral posterior wall.

Surgical treatment. Surgical approach selection was made taking into account the location of the tumor, the prognosis and the necessity for stabilization.

C1-C2 region can be approached mainly in 4 ways:

- transoral

- retropharyngian submandibular antero-lateral

- postero-lateral with C2 articular resection

- posterior with C1/C2 laminectomy +/-suboccipital craniectomy

In one pacient we resected the odontoid tumor by posterior approach with suboccipital craniectomy. Tumor resection was made transdurally.

For subdural and intramedulary tumors surgical approach was posterior with $\mathrm{C} 1 / \mathrm{C} 2$ laminectomy and if necessary suboccipital craniectomy or $\mathrm{C} 2 / \mathrm{C} 3$ articular resection for intraforaminal intra/extradural dumpbell tumors. $(10,13)$

Stabilization of the cranio-spinal junction was made according to the estimated life expectancy of the patient. In patients with 
aggresive lesions and poor prognosis a combined synthesis was usually obtained (methylmethacrylate anterior and occiput-C4 metallic synthesis posterior) which allowed for rapid mobilization and reduced the necessity of external immobilisation with a halo fixator. $(6,7,8,9)$

\section{Results}

All four types of approaches were used to address the craniospinal junction tumors.

51 surgical interventions were done on 44 pacients (7 patients were operated both by anterior and posterior approach).

6 patients were operated only through antero-lateral retropharyngeal approach, for resection of anterior located vertebral tumors, with $\mathrm{C} 2$ body and odontoid proces reconstruction with methylmethacrylate.

Out of 31 posterior approaches (posterolateral or posterior)

- 7 intramedullary tumors were approached through myelotomy on the midline or on the posterior median sulcus.

- 11 were posterior approaches for subdural extramedullary tumors, including dumpbell tumors.

- For 11 cases of tumors that involved the posterior arch of $\mathrm{C} 1$ and $\mathrm{C} 2, \mathrm{C} 1 / \mathrm{C} 2$ tumoral dislocations or cases where only occipito-cervical fixation was advocated, we used a posterior approach.

- 2 cases of epidural tumors were approached also by posterior approach

In 7 cases we used both an anterior and posterior approach in two separate surgical interventions, usually using the anterior approach as the first surgical procedure (tumor resection and C2 body reconstruction), followed by posterior rachisynthesis. $(12,13,14)$

For 1 patient the first procedure was a biopsy and transoral C2 vertebroplasty. (7)
For 13 patients (6 with anterior approach only and 7 with combined anterior and posterior approaches) we used methylmethacrylate thruough an anterior approach, with contentions in the dens, C3 and $\mathrm{C} 4$ vertebral bodies. We preferred methylmethacrylate for reconstruction (figure 1) because it allows patient mobilisation immediately postoperatively especially usefull in patients with a low life expectancy. It is a relatively simple method with low costs. $(8,12)$

\section{STAGES OF SURGERY}
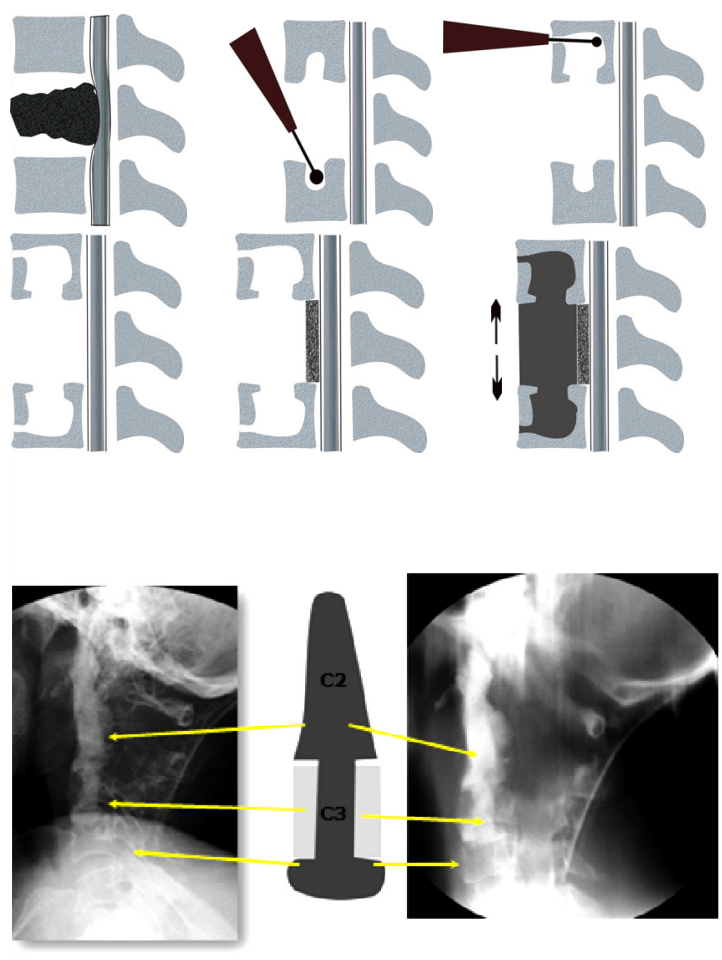

Figure 1 Drilling the retention cavities for vertebral reconstruction with methylmethacrylate 
Posterior synthesis was needed in 10 pacients. In most of the cases (6 cases) with posterior fixation the procedure combined bony and metallic material (OASIS system picture 1). In 4 cases with occipitocervical synthesis only bone graft from the 12th rib or/and iliac crest was used. The bone graft was fixed in place with metal wires, screws and plates. All these 10 pacients presented clinical signs of occipito-cervical instability preoperatively that remitted postoperatively.

\section{Clinical results}

There was a neurological improvement in 17 patients with a gain on ASIA scale in the first postoperative week by a mean of 8 points, while 9 patients worsened immediately postoperatively with a mean decrease on ASIA score of 10 points (evaluation at 24 hours postop). Of the latter 9 patients, 6 patients improved neurologically at 7 days postoperatively, with an almost complete remission of the deficits that had appeared postoperatively. In one patient (intramedullary tumor) with a sustained worsening, the deficits partially improved after 2 months of rehabilitation. Two patients died.

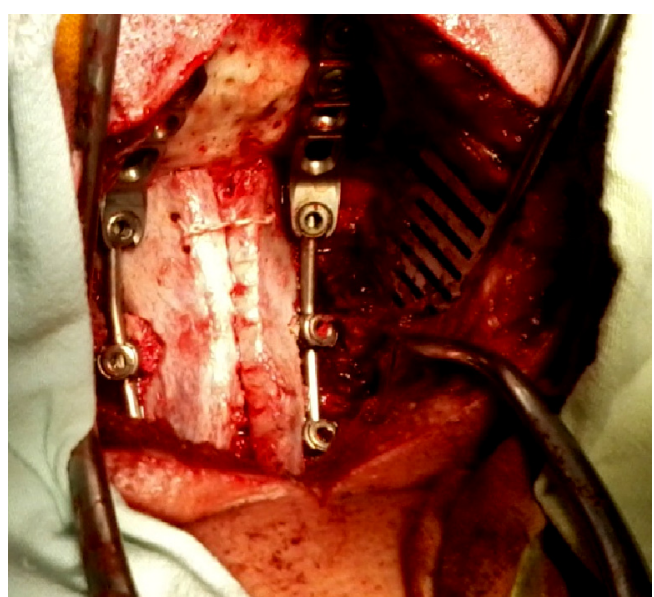

Picture 1 Occipito-cervical combined bony and metal fixation and OASIS system
There were no neurological deficits either preoperatively or postoperatively in 18 patients. The pain due to occipitocervical instability disappeared postoperatively in all the cases.

There was no intraoperative mortality in this series, but 2 patients in whom the tumor extended to the brainstem died in the $3^{\text {rd }}$ respectively 16 th day postoperative.

- Frankel A: 0

- Frankel B: 0

- Frankel C: 9

- Frankel D: 18

- Frankel E: 10

- Sindrom radicular: 5
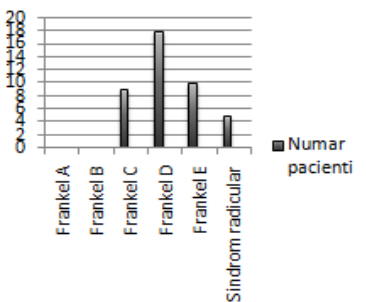

Figure 2 Preoperative neurological status

\section{Illustrative cases}

CASE 1. A 58 year old female (picture 2) who presented with occipitocervical pain with the characteristic of instability.
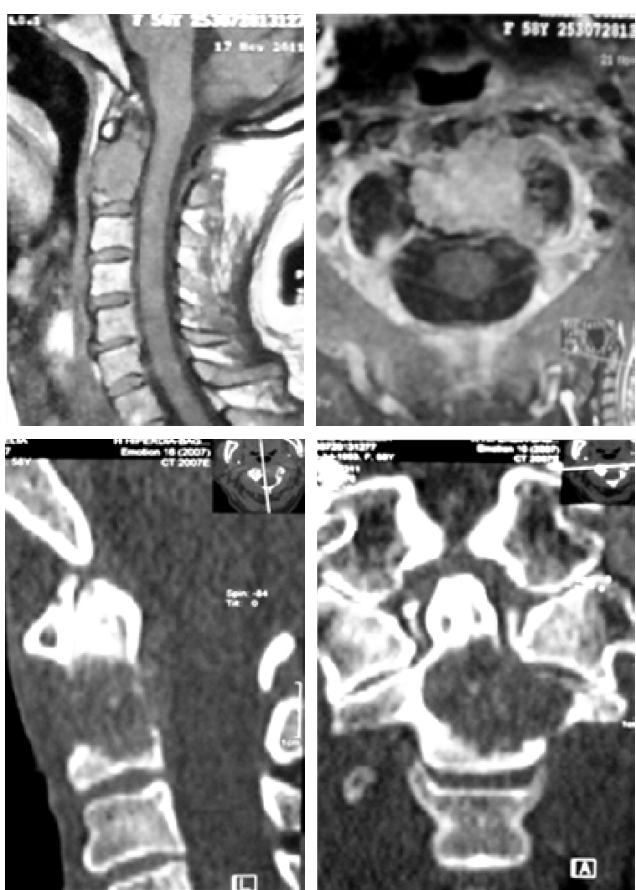

Picture 2 Sagital and axial T1 MRI and vertebral CT reconstructions in sagital and frontal planes 
The MRI and vertebral C1 and C2 exam showed a C2 vertebral body tumor that involved the left $\mathrm{C} 2$ lateral mass with no medullary compression at this level.

An antero-lateral retropharyngeal approach was used, the tumor was resected and the C2 vertebral body was reconstructed with methylmethacrylate (picture 3).

The patient presented no postoperative pain and the spine was stable and aligned. The histopathologic exam showed uterine carcinoma metastasis and the pacient was sent for oncologic treatment.

CASE 2. A 44 year old male (picture 4) that presented right occipital pain with a radicular character of Arnold neuralgia, accentuated on head movement. CT and MRI scans showed a tumor that involved the $1 / 3$ right lateral anterior $\mathrm{C} 1 \mathrm{arch}$, right C1 lateral mass and right half of posterior arch of C1.

We decided for open surgery with histopathological diagnostic purposes and $\mathrm{C} 2$ right root decompression and also for occiput-C3 and C4 fixation with OASIS system (picture 5) to ensure stability. This patient had no instability or occipital neuralgia postoperatively. The histopathological exam revealed osteoblastoma.

CASE 3. A 27 year old woman admitted for upper cervical pain. A dens and C2 body tumor is shown on CT and MRI scans (picture 6).

We decided for combined surgery using both an anterior and a posterior approach and $\mathrm{C} 2$ reconstruction with cement, with retention in $\mathrm{C} 3$ body. The pacient was immobilised in a halo and in a second stage surgery we performed a posterior approach for C1 and C2 sublaminar wires (picture 7). Histopathology: thyroid adenocarcinoma metastasis.

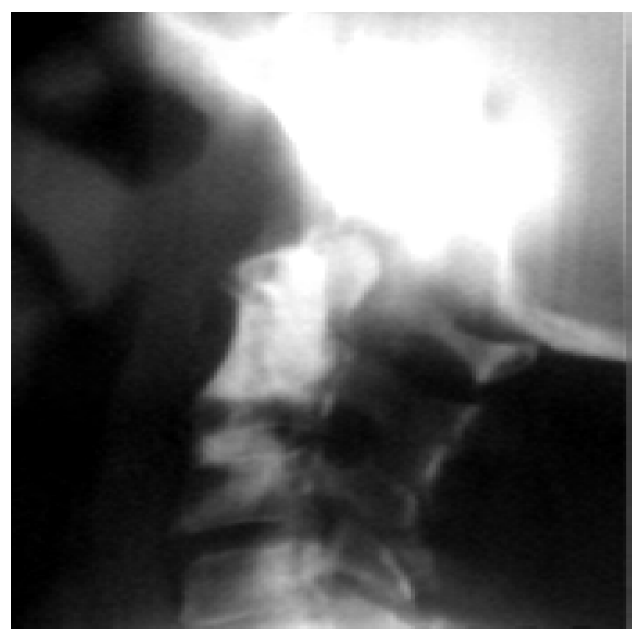

Picture 3 Latero-lateral X-ray postoperative image
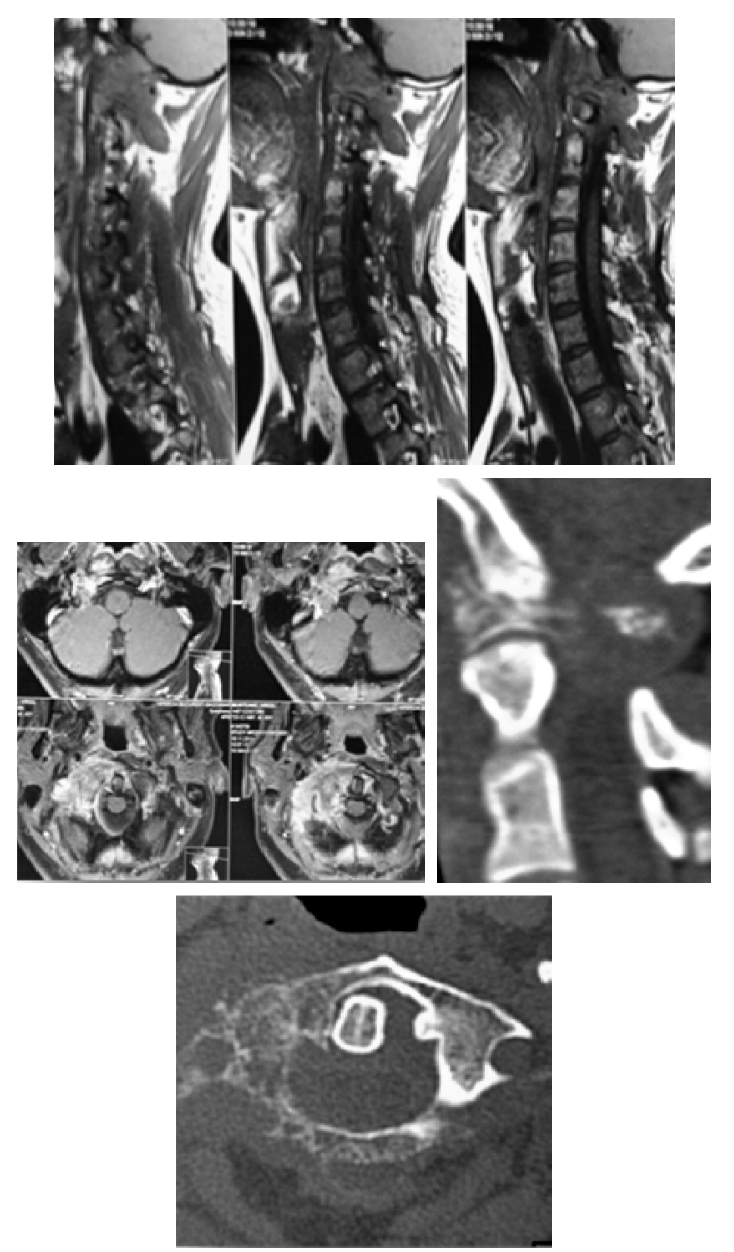

Picture 4 Sagital and axial T1MRI and CT - C1 tumor 


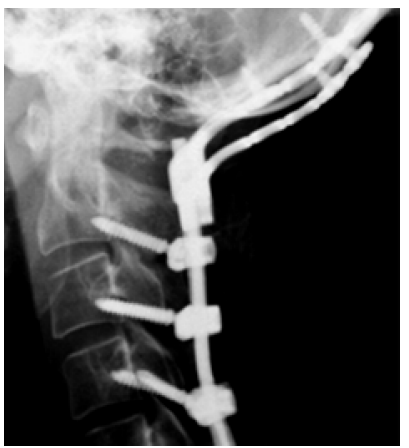

Picture 5 Postoperative occipito-cervical Xraycontrol
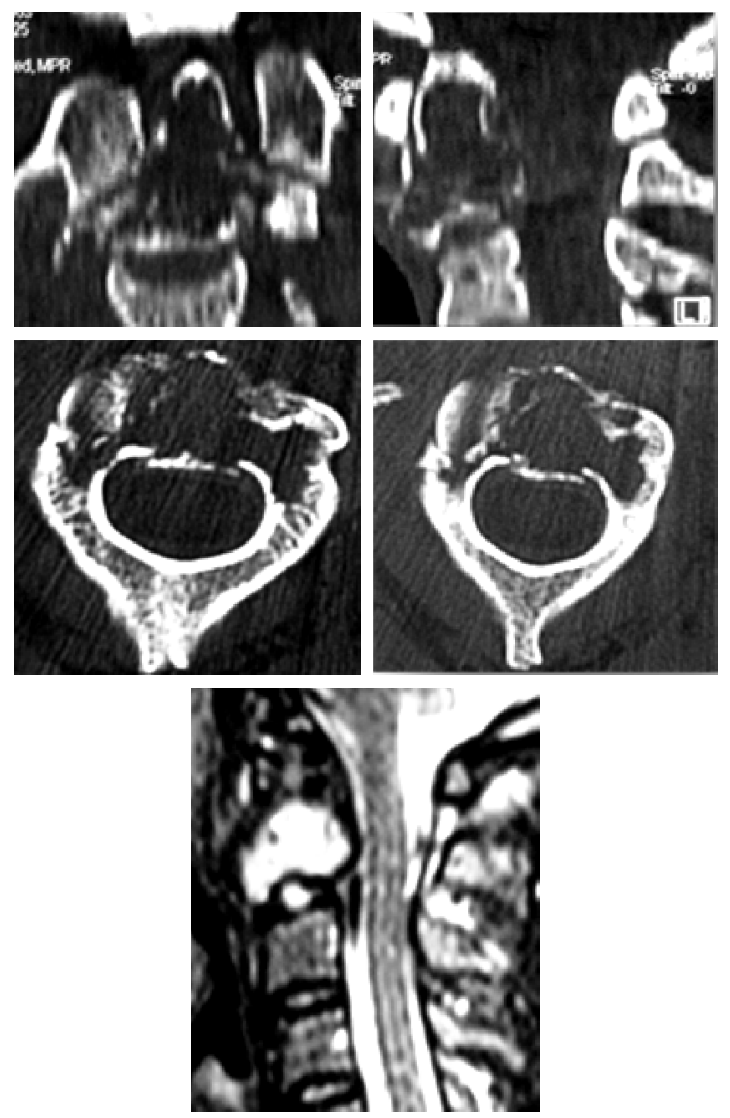

Picture 6 Preoperative frontal, sagital and axial CT showing $\mathrm{C} 2$ vertebral posterior wall destruction.

Sagital T2 MRI showing the tumor

CASE 4. A 49 year old female with upper cervical pain, occipitocervical instability and $\mathrm{C} 2$ root irritation syndrome (picture 8). We decided for a transoral biopsy (breast cancer metastasis) and C2 vertebroplasty.

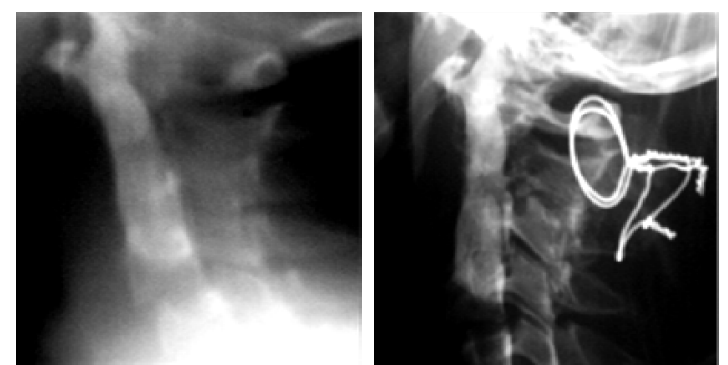

Picture 7 Appearance after initial surgery and after C1-C2 posterior wire fixation
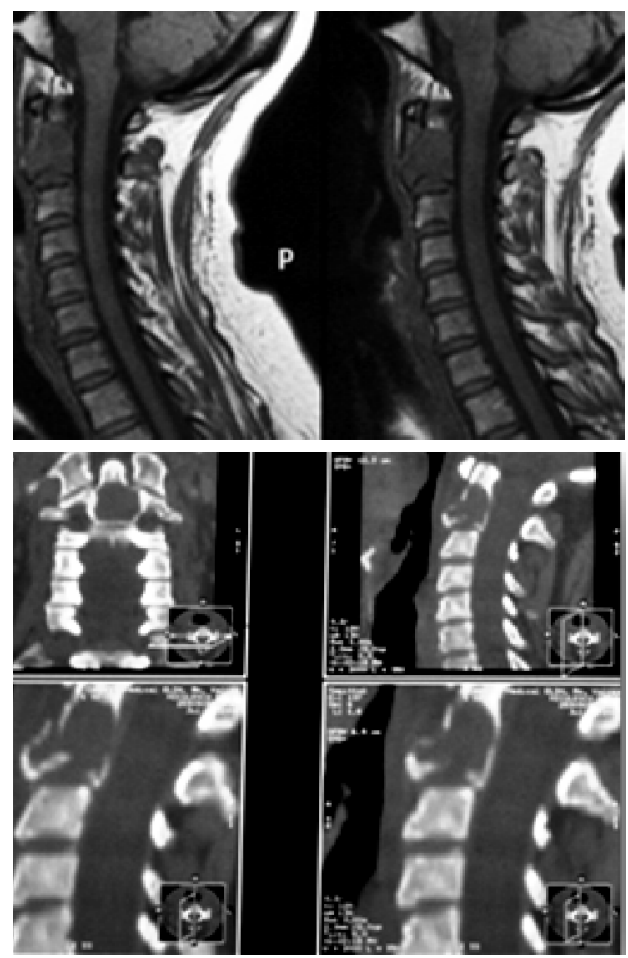

Picture $8 \mathrm{C} 2$ vertebral lysis in the midline, preserving the posterior wall. (Sagital T2 MRI, Frontal and sagital CT)

Following the first intervention the pain due to instability didn't disappear and we decided for an occipitocervical fixation with OASIS (picture 9) and following this second surgery the pain was alleviated.

CASE 5. A 68 year old male admitted for incomplete tetraplegia Frankel D with high cervical level and predominantly right side deficits. A right "dumpbell" C1-C2 tumor originating in the $\mathrm{C} 2$ root at the level of the 
C1-C2 foramina with intra and extrarahidian extension (picture 10). The preoperative planning also evaluated the tumor - vertebral artery relationship using angio CT and angiography that showed a marked right vertebral artery caliber reduction.

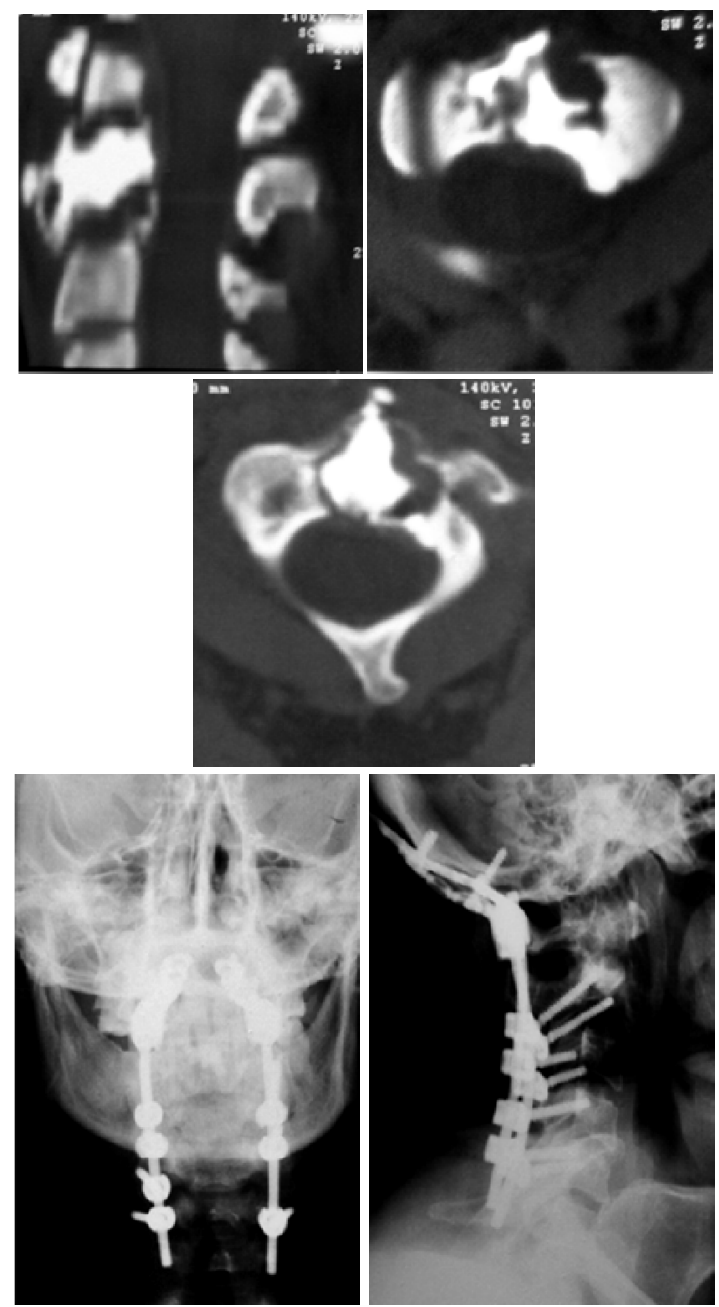

Picture 9 Postoperative image following transoral vertebroplasty (sagital and axial control CT) and posterior fixation with OASIS system (postoperative X-ray control).
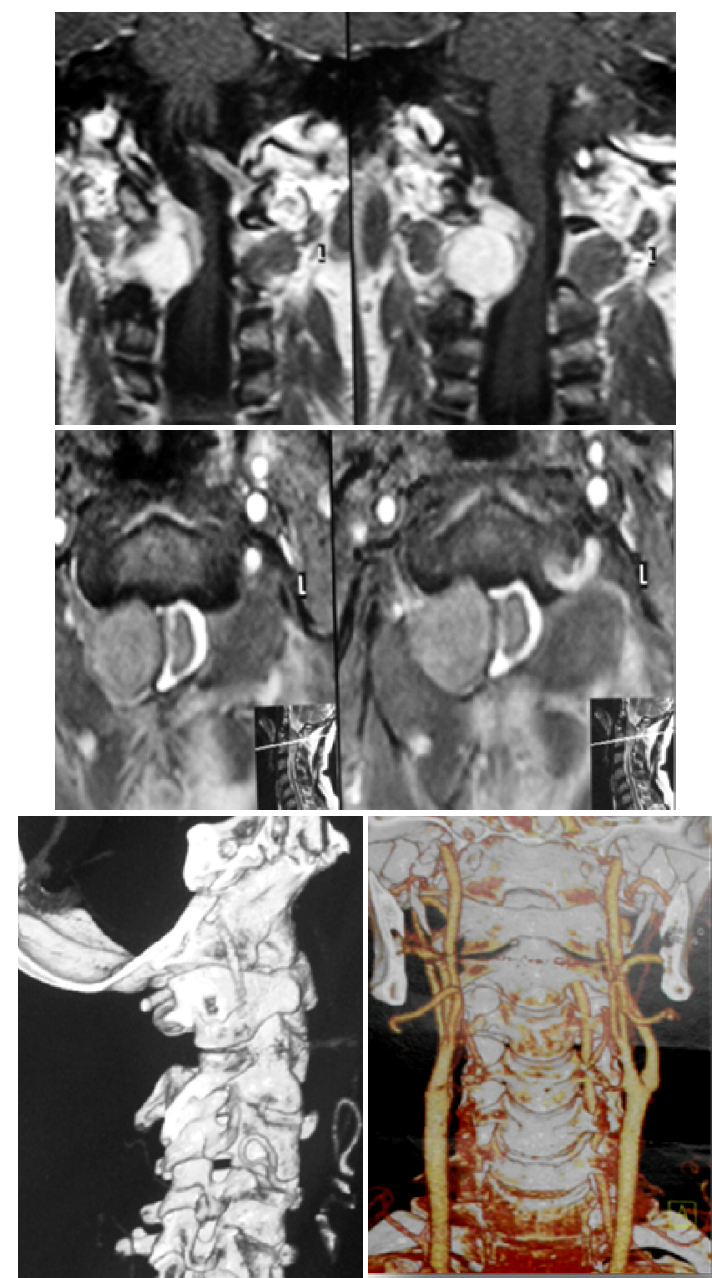

Picture 10 "Dumpbell" C1-C2 right tumor compressive on the right vertebral artery. Frontal T1

MRI, axial T2 MRI, and cervical angio CT.

The tumor was resected through a posterior approach with $\mathrm{C} 1$ and $\mathrm{C} 2$ laminectomies and right sided articular resections. Histopathological examination schwannoma.

CASE 6. A 58 year old woman admitted with Frankel C high cervical level incomplete tetraplegia and a preoperative ASIA score of 70 points. The MRI shows a subdural tumor with right anterolateral extramedullary extension (picture 11). A posterior approach with $\mathrm{C} 1, \mathrm{C} 2$, and C3 laminectomy was advocated and slightly 
enlarged on the right side. An arched incision was used in the dura concave to the right. The posterior C2 root was sectioned. We found a solid tumor inserted on the right anterolateral dura and we managed a total resection and coagulation of the base of dural insertion (Simpson 1).

The patient improved significantly postoperatively and the histopathological result was meningioma.

CASE 7. A 36 year old male admitted with Frankel $C$ high cervical level incomplete tetraplegia and progressive onset of symptoms. The MRI showed a tumor at the junction between the brainstem and the medulla, descending to C3, that presented two polar cysts, of which the cranial one was smaller (picture 12). We used a midline myelotomy and the tumor was completely resected.
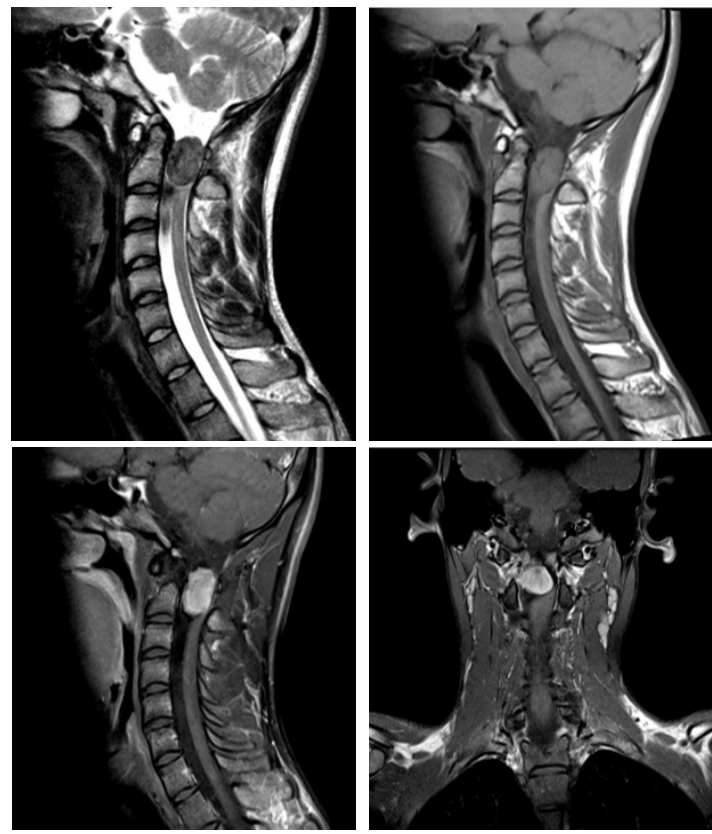
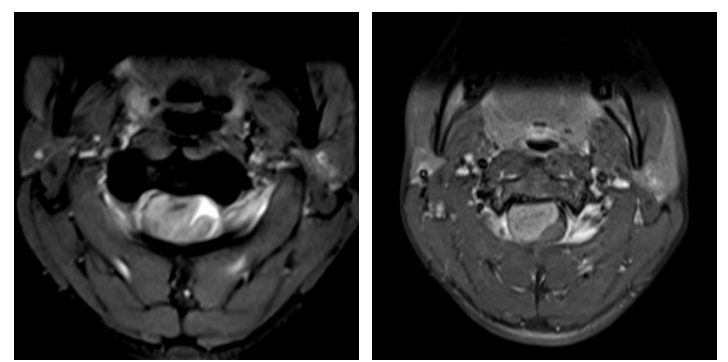

Picture 11 Sagital, frontal and axial MRI showing a subdural extramedullary C2 tumor
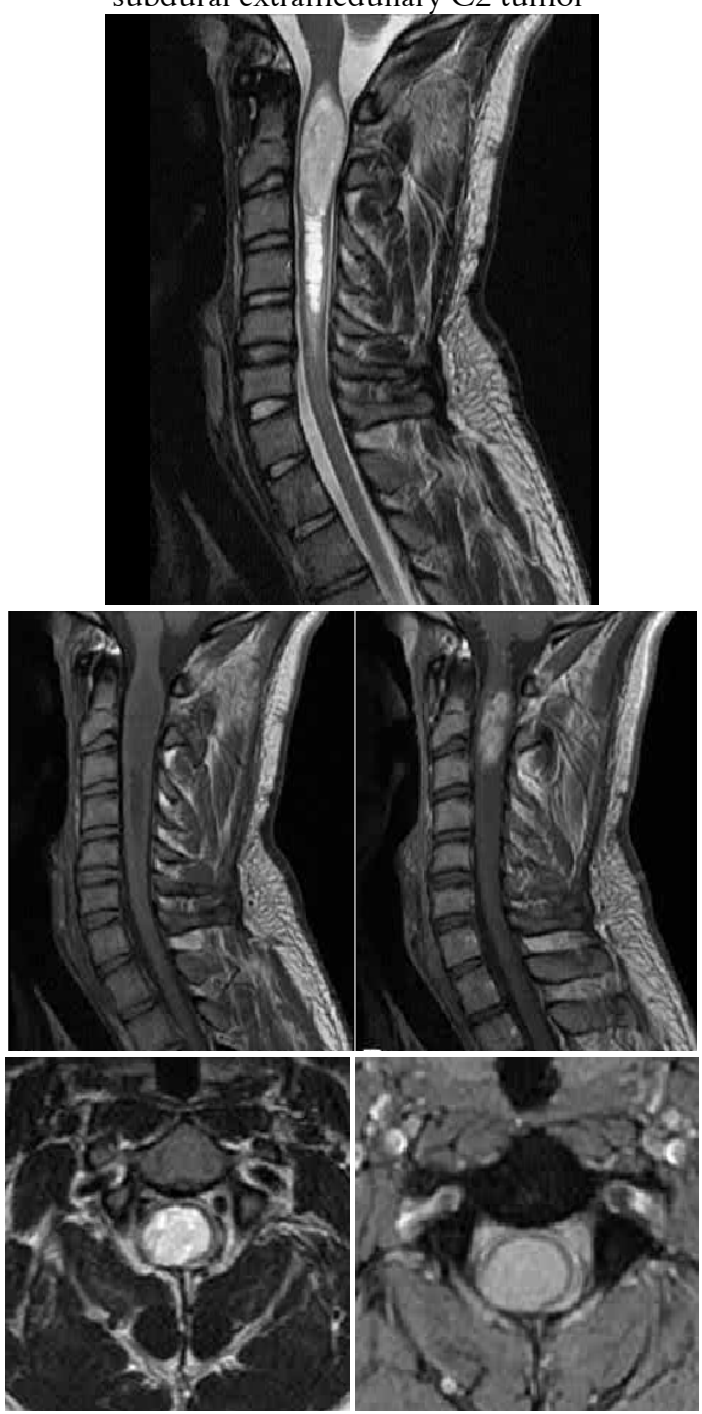

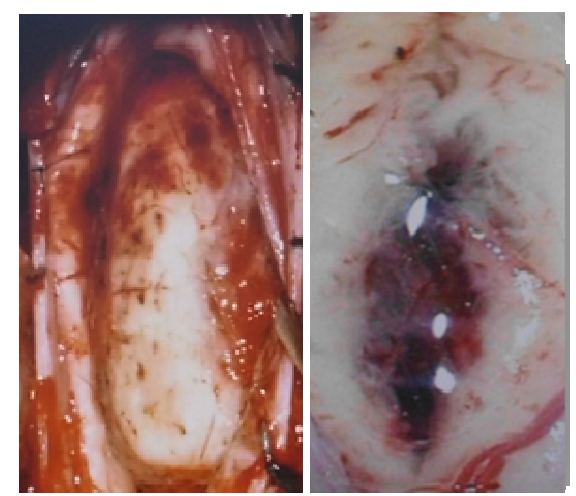

Picture 12 T2, T1 sagital and axial MRI showing intramedullary tumor with polar cysts; and intraoperative aspect.

The patient deteriorated postoperatively from 80 to 62 points on ASIA score, but subsequently he improved in the rehabilitation department.

\section{Discussions}

The use of imaging investigations (CT and MRI) and the development of fixation methods allow diagnosis and radical resection of cranio-spinal junctional tumors while preserving stability. Nevertheless, the surgical treatment for cranio-spinal junctional tumors challenges the neurosurgeon with many difficulties due to the less frequent tumoral pathology at this level and the unique biomechanical characteristics of the region.

\section{Surgical decision}

For subdural or intramedullary tumors the decision over which approach should be used is not difficult. Moreover the surgical success depends on the location, extension and nature of the tumor as well as on the neurosurgeon's experience.

Difficulties are seen in case of tumors that involve the bone and ligaments at the C1-C2 level. Decisions over approach and stability methods are essential.

The chosen surgical approach should ensure tumor resection or at least tumor tissue biopsy for the histopathological exam. It must also take into account the tumor location and the patient's prognosis. Patient survival is directly related to the extent of the resection and the histopathological type of tumor. If both anterior and posterior approaches are needed then more than one surgery intervention (usually two) are prefered.

Submandibular retropharyngian approach exposes the anterior cervical spine from the level of the cranio-spinal junction to the $\mathrm{C} 4$ level on the median line. For a better exposure the facial nerve may be dissected and the submandibulary salivary gland may be reflected. This approach has advantages over the transoral approach because it has no communication with the oral cavity and it may attain concomitent stabilisation of the anterior spine with bone graft or with methylmethacrylate. The disadvantages are the depth of the operating field, the long dissection time, compression on the nerves IX, XII and on the pharynx with possible paresis of these nerves and postoperatory deglutition impairment (usually transient). (9, 13, 14)

Postero-lateral and posterior approach is used for tumors that affect the $\mathrm{C} 1 / \mathrm{C} 2$ posterior arch, dumpbell tumors, extramedullary subdural tumors and intramedullary tumors. The advantages of this approach are that it has become familiar to many neurosurgeons as it is frequently used and it allows posterior rahisynthesis with bone grafts or metallic fixation. $(5,6$, $8,9)$

\section{Craniospinal junction stabilisation}

Most tumors that involve the craniospinal junction, especially the axis, lead to instability. 
Fusion techniques have been chosen according to the nature and location of the tumor, the degree of bone involvement and the patient's prognosis.

The most widely used procedure in obtaining craniospinal junction stability has been the posterior occipito-cervical fusion, usually after anterior tumoral resection with or without anterior reconstruction with methylmethacrylate. Frequently the metallic fixation has been accompanied by bone graft placement. The bone graft was harvested during the same operating procedure from the iliac crest or from a rib. Postoperatively patients have been immobilised with a halo for 4 to 8 weeks. Fusions between C1/C2 posterior arches need a shorter immobilisation period, usually with a neck collar.

Patients with a poor prognosis and those who need radiotherapy after surgery need a combined solid fixation (anterior with methylmethacrylate and posterior synthesis occiput-C4) that allows a rapid mobilisation and reduces the necessity of external halo immobilisation.

\section{Complications}

There were no intraoperative fatalities in this series.

Two patients with intramedullary tumors with intracranian extension died on the $3 \mathrm{rd}$ and on the 16th day postoperatively.

7 patients worsened postoperatively with a median decrease in ASIA score of 10 points, however 6 of these were better after the first week with an almost complete recovery of the deficits.

2 pacients with anterior approaches and C2 vertebral reconstruction with methyl methacrylate presented dysphagia, dysphonia and shortness of breath postoperatively. The follow up plain $\mathrm{x}$-ray showed fractures in the acrylic cement with consequent $\mathrm{C} 1 / \mathrm{C} 2$ luxation. At reintervention, on anterior approach, the cement was excised and solid retentions were done in C3 and C4 vertebral bodies and the anterior fixation was repeated. After a halo immobilization the patients were operated through posterior approach for occipito-cervical fixation.

CSF fistula was present in 2 cases, but it eventually closed spontaneously after continuous lumbar drainage.

It is important to have a good selection of patients in order to keep the surgical morbidity at low rates.

\section{Conclusions}

Surgery is a viable but not at all simple solution for the cranio-spinal junction tumors. It allows decompression of vital nerve structures and stabilisation of the cranio-spinal junction, total or partial tumor removal, histopathological diagnosis and improves the quality of life. The type of approach depends on the tumor location and the success of surgery depends mainly on the knowledge and experience of the neurosurgeon.

The complete tumor resection has to decompress the medulla without producing any lesions to vital vascular and neural structures in this area. The surgical technique used must give stability to the cranio-spinal junction. The need for combined approaches is relatively frequent, for tumor resection and decompression as well as for stabilisation of the occipitocervical area.

\section{References}

1.Bortoluzzi M: Neoplasms of spinal origin involving the foramen magnum. Neurodiagnostic considerations on 36 cases. J Neurosurg Sci 24:71-76, 1980.

2.Boriani S, Weinstein JN, Biagini R: Primary bone tumors of the spine. Terminology and surgical staging. 
DOI: $10.2478 / v 10282-012-0012-3$

Spine 22:1036-1044, 1997.

3.Bilsky MH, Shannon FJ, Sheppard S, Prabhu V, Boland PJ: Diagnosis and management of a metastatic tumor in the atlantoaxial spine. Spine 27:1062-1069, 2002.

4.Buckingham MJ, Tew JM Jr, Wiot JG: The diagnosis and surgical treatment of craniocervical junction tumors, in Camins MB, O'Leary PF (eds): Disorders of the Cervical Spine. Baltimore: Williams \& Wilkins, 1990, pp 497-506.

5.Clark CT, Apuzzo MLJ: The evaluation and management of trauma to the odontoid process, in Cooper PR (ed): Management of Posttraumatic Spinal Instability. Park Ridge, Ill: American Association of Neurological Surgeons, 1990.

6.Deen HG, Birch BD, Wharen RE, Reimer R: Lateral mass screw-rod fixation of the cervical spine: a prospective clinical series with 1-year follow-up. Spine J 3:489-495, 2003.

7.Hadley MN, Spetzler RF, Sonntag VKH: The transoral approach to the superior cervical spine. A review of 53 cases of extradural cervicomedullary compression. J Neurosurg 71: 16-23, 1989.
8.Jackson RJ, Gokaslan ZL: Occipitocervicothoracic fixation for spinal instability in patients with neoplastic processes. J Neurosurg (Spine 1) 91:81-89, 1999.

9.John G. Piper, Arnold H. Menezes, Management strategies for tumors of the axis vertebra - J Neurosurg 84:543-551, 1996.

10.Jörg Klekamp, Madjid Samii: Surgery of Spinal Tumors ISBN 978-3-540-44714-6 Springer Berlin Heidelberg New York.

11.Marino R: International Standards for the Neurological Classification of Spinal Cord Injury. Chicago: American Spinal Injury Association, 2000.

12.Menezes AH, Traynelis VC: Tumors of the craniovertebral junction, in Youmans JR (ed): Neurological Surgery, ed 4.

13.Watkins RG, Dillin WH: Surgical approaches to the cervical spine, in Camins MB, O'Leary PF (eds): Disorders of the Cervical Spine. Baltimore: Williams \& Wilkins, 1990.

14.Whitesides TE Jr: Lateral retropharyngeal approach to the upper cervical spine, in The Cervical Spine Research Society Editorial Committee (eds): The Cervical Spine, ed 2. Philadelphia: JB Lippincott, 1989. 